Georgia State University

ScholarWorks @ Georgia State University

\title{
An examination of the factorial structure of the Schizotypal Personality Questionnaire-Brief (SPQ-B) among undergraduate students
}

\author{
Michael T. Compton \\ Emory University School of Medicine, Department of Psychiatry and Behavioral Sciences \\ Sandra M. Goulding \\ Emory University School of Medicine, Department of Psychiatry and Behavioral Sciences \\ Roger Bakeman \\ Georgia State University, bakeman@gsu.edu \\ Erin B. McClure-Tone \\ Georgia State University, Department of Psychology, etone@gsu.edu
}

Follow this and additional works at: https://scholarworks.gsu.edu/psych_facpub

Part of the Psychology Commons

\section{Recommended Citation}

Compton, Michael T.; Goulding, Sandra M.; Bakeman, Roger; and McClure-Tone, Erin B., "An examination of the factorial structure of the Schizotypal Personality Questionnaire-Brief (SPQ-B) among undergraduate students" (2009). Psychology Faculty Publications. 130.

https://scholarworks.gsu.edu/psych_facpub/130

This Article is brought to you for free and open access by the Department of Psychology at ScholarWorks @ Georgia State University. It has been accepted for inclusion in Psychology Faculty Publications by an authorized administrator of ScholarWorks @ Georgia State University. For more information, please contact scholarworks@gsu.edu. 


\section{Brief Report}

\section{An examination of the factorial structure of the Schizotypal Personality Questionnaire- Brief (SPQ-B) among undergraduate students}

Michael T. Compton, M.D., M.P.H. ${ }^{\text {a }}$

Sandra M. Goulding, M.P.H. ${ }^{\text {a }}$

Roger Bakeman, Ph.D. ${ }^{\text {b }}$

Erin B. McClure-Tone, Ph.D. ${ }^{\text {b }}$

${ }^{a}$ Emory University School of Medicine, Department of Psychiatry and Behavioral Sciences, Atlanta, Georgia, USA, 30322

${ }^{\text {b }}$ Georgia State University, Department of Psychology, Atlanta, Georgia, USA, 30302

\section{Corresponding Author}

Michael T. Compton, M.D., M.P.H.

Emory University School of Medicine

Department of Psychiatry and Behavioral Sciences

49 Jesse Hill Jr. Drive, S.E., Room \#333

Atlanta, GA 30303

TEL: 404-778-1486 / FAX: 404-616-3241

E-MAIL: Michael.Compton@emory.edu 


\begin{abstract}
Cognitive-perceptual, interpersonal, and disorganized subscales of the Schizotypal Personality Questionnaire-Brief (SPQ-B), reflecting the three commonly used subscales of the full-version SPQ, have been used in a number of studies. However, the factorial validity of SPQB subscales remains to be clarified. Utilizing data from 825 undergraduate students, confirmatory factor analyses involving the 22 items of the SPQ-B were conducted. A significant $\chi^{2}$ difference test favored the 3-factor over the 1-factor model and fit indices for the 3-factor model were generally satisfactory. However, several of the items may index more than one of the hypothesized factors, so the item-factor separation is not sharp. Thus, more research is needed on the factorial validity of the increasingly used SPQ-B subscales.
\end{abstract}

Key Words: Confirmatory factor analysis; Schizotypal Personality Questionnaire; Schizotypy 


\section{Introduction}

Schizotypy, a multidimensional behavioral phenotype, is of interest to schizophrenia researchers for several reasons. For example, it is associated with neurocognitive and social deficits present in schizophrenia (Dickey et al., 2005; Gooding et al., 2006). Schizotypy is also associated with risk for developing schizophrenia (Miller et al., 2002; Tyrka et al., 1995). Additionally, some studies indicate that schizotypy may be more common in biological relatives of individuals with schizophrenia than in other non-psychiatric samples (Kendler et al., 1995; Kremen et al., 1998), though it should be noted that some studies comparing the rates of schizotypal traits in relatives of patients with schizophrenia and in relatives of patients with affective psychoses have not confirmed the specificity of schizotypy to schizophrenia (Coryell and Zimmerman, 1989; Gilvarry et al., 2001; Mata et al., 2003; Squires-Wheeler et al., 1988; Squires-Wheeler et al., 1989). Although interview-based instruments are occasionally used, the measurement of schizotypy usually relies on at least one of many self-report inventories. Such self-report instruments generally use “yes/no" or "true/false” formats and include 30-100 items; however, shorter, more time-efficient measures of the complex schizotypy construct have been proposed.

The abbreviated or brief version of the Schizotypal Personality Questionnaire (SPQ-B; Raine and Benishay, 1995), a 22-item self-report measure requiring approximately two minutes to complete, has been used in a number of studies pertaining to either schizotypal personality disorder or dimensional schizotypy in non-clinical samples, predominantly involving undergraduate college students (Aycicegi et al., 2005; Bailey and Swallow, 2004; Bedwell and Donnelly, 2005; Bedwell et al., 2006; Houran et al., 2001; Jahshan and Sergi, 2007; Mata et al., 2005; Schiffman et al., 2005). Although an extensive body of literature has examined the 
factorial structure of the full 74-item SPQ (with three subscales commonly derived to assess cognitive-perceptual, interpersonal, and disorganized domains of schizotypy, though others have documented a 4-factor structure; Compton et al. 2009), considerably less is known about the factorial validity of the SPQ-B. Given prior data (Compton et al., 2007) indicating that a singlefactor solution of the SPQ-B may provide equal fit compared to the 3-factor solution used in some studies (e.g., Bailey and Swallow, 2004; Bedwell and Donnelly, 2005; Bedwell et al., 2006; Schiffman et al., 2005), a confirmatory factor analysis was conducted in an independent, larger sample, to examine the factorial structure of the 22 items of the SPQ-B. In another report (Compton et al., 2009), data from this same sample were used to determine the best-fitting factor structure of the nine subscales of the full 74-item SPQ from among five models that have been proposed in the literature, as well as five additional hierarchically related models. The present report focuses on the SPQ-B and its proposed subscales given their increasing use in research despite insufficiently studied psychometric properties.

\section{Methods}

This study relied on a sample of 825 undergraduate college students attending an urban state university in the southeastern United States. The mean ( \pm standard deviation) age of participants was $20.1 \pm 1.7$ years (range: 18-26 years). Seventy-seven percent of participants were female, $46 \%$ were single and not dating anyone regularly, and $47 \%$ were in their freshman year. In terms of the racial distribution, $45 \%$ of the sample described themselves as White/Caucasian, 31\% as Black/African American, 10\% as Asian American, 6\% as bi-racial, and 5\% as Latino/Hispanic. Students were invited to participate through a recruitment statement on an online program used to manage the undergraduate research pool at the university. Participants read an online consent form approved by the university's Institutional Review Board before 
proceeding to the survey, with survey completion implying their consent for the researchers to use their data.

As part of the larger survey, participants completed the full 74-item version of the Schizotypal Personality Questionnaire (SPQ, Raine, 1991; Raine et al., 1994). The SPQ was designed to assess all nine diagnostic criteria for schizotypal personality disorder (American Psychiatric Association, 1980). Each “yes” response counts as one point, with total scores ranging from 0 to 74. Scores for the three domains of schizotypy are derived by simple summation of relevant subscale raw scores (the cognitive-perceptual domain includes the ideas of reference, odd beliefs or magical thinking, unusual perceptual experiences, and paranoid ideation/suspiciousness subscales; the interpersonal domain includes the excessive social anxiety, no close friends, constricted affect, and paranoid ideation/suspiciousness subscales; and the disorganized domain includes the odd or eccentric behavior and odd speech subscales). The 22-item SPQ-B (Raine and Benishay, 1995) was developed using the most reliable items from the SPQ. Thus, this abbreviated instrument constitutes a subset of the SPQ items. The nine subscale scores typically generated for the SPQ cannot be derived from the SPQ-B due to the restricted number of items. As mentioned above, however, some researchers have derived three domains scores (cognitive-perceptual, 8 items; interpersonal, 8 items; and disorganized, 6 items), in addition to a total score from the SPQ-B.

Descriptive statistics, internal consistency reliability (Cronbach's $\alpha$ ) coefficients, and correlations among the three subscale scores were computed using SPSS 15.0. The Linear Structural Relations Program (LISREL 8.7) was used for confirmatory factor analyses. As in the prior SPQ confirmatory factor analysis study (Compton et al., 2007), two confirmatory factor analyses of the SPQ-B were conducted to determine whether or not the 3-factor solution provides 
better fit to the data than a single-factor solution. Several indices were selected $a$ priori to assess

the fit of the measurement model to the data: (1) the normed model chi-square $\left(\chi^{2}{ }_{M} / \mathrm{df}_{\mathrm{M}}\right)$, (2) the Steiger-Lind root mean square error of approximation (RMSEA) and its 90\% confidence interval (CI), (3) the standardized root mean square residual (SRMR), and (4) the Bentler comparative fit index (CFI). To compare hierarchical (nested) models, the chi-square difference $\left(\chi^{2}{ }_{D}\right)$ test was used, in which the $\chi^{2}{ }_{\mathrm{M}}$ for the trimmed model is subtracted from that of the initial model; the $d f$ for $\chi^{2}{ }_{D}$ is the difference in $d f$ between the initial and trimmed models. To enhance precision of results, all LISREL analyses were run using very accurate input data from covariance matrices (i.e., values with five significant digits).

\section{Results}

In this sample, the mean total SPQ-B score was 7.5 \pm 4.9 . Cognitive-perceptual,

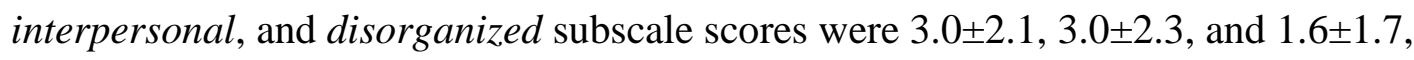
respectively. It is noteworthy that all of these mean scores are lower than those reported by Raine and Benishay (1995), in their sample of 220 undergraduate students (9.6 $\pm 5.3,3.6 \pm 2.3,3.6 \pm 2.4$, $2.5 \pm 1.9$, respectively). The Cronbach's $\alpha$ internal consistency reliability coefficient for the total SPQ-B score was .84. The $\alpha$ coefficient values for the cognitive-perceptual, interpersonal, and disorganized subscales were .69, .77, and .72, respectively. The cognitive-perceptual score was significantly correlated with the interpersonal score $(r=.39)$ and the disorganized score $(r=.48)$, and the latter two scores were correlated as well $(r=.51$; all $p<.001)$.

Both the single-factor and 3-factor models for the 22 items of the SPQ-B were examined using LISREL. Results of these confirmatory factor analyses are given in Table 1. The singlefactor model failed to meet key criteria for good fit. The 3-factor model fit significantly better than the single-factor model, $\chi^{2}{ }_{\mathrm{D}}(3, N=825)=899, p<.001$. Even so, its fit was not completely 
satisfactory. Specifically, using criteria from Kline (2005), for the 3-factor but not the singlefactor model, its normed $\chi^{2}$ was $<5$ ( $<3$ would be better) and its RMSEA was $<.08$, suggesting a reasonable error of approximation if not close approximate fit (which requires $<.05$ ). For both, the top 90\% CI did not exceed .10; nor did the SRMR (values <.10 are regarded as favorable). However neither CFI exceeded .90, a value regarded as indicative of reasonably good fit (assuming the base model is plausible, which is arguable; see Kline, 2005). Guided by modification indices and given these data, a model with a normed $\chi^{2}<3$, a SRMSA less than .05 , and a CFI greater than .90 requires that 11 of these 22 items load on more than one factor, which suggests some overlap between items and factors (recall the correlations between factor scores reported earlier).

These finding did not change meaningfully when males and females were considered separately. Cronbach's $\alpha$ values for the three factors were similar for both, and a multi-group structural equation modeling analysis showed no significant change in fit when factor loadings were constrained to be the same for males and females.

\section{Discussion}

The three-factor model of the SPQ-B, upon which use of the cognitive-perceptual, interpersonal, and disorganized subscales is based, did perform better than the one-factor model in this sample, but the one-to-one correspondence between each factor and its items was questionable, given the somewhat marginal fit of the 3-factor model. Thus, despite the significant $\chi^{2}$ difference test appearing to favor the 3-factor over the 1-factor model, the overall fit indices were marginal, leading to the conclusion that evidence of a three-factor model with the 22 items is reasonable, but not overwhelming. Several of these items may index more than one of the hypothesized factors, so the item-factor separation is not sharp. Although the SPQ-B was 
designed for use in large-scale screening for schizotypal personality disorder prior to a confirmatory clinical interview and for research on the correlates of schizotypy in the normal population (Raine and Benishay, 1995), the factorial validity of its three proposed subscales was not unequivocally demonstrated in the present sample. Thus, findings from the present analysis may argue against assuming established factorial validity of very brief SPQ-B subscales across samples.

At least three studies have conducted exploratory factor analyses of the 22 items of the SPQ-B. Among adolescent psychiatric inpatients, Axelrod and colleagues (2001) demonstrated that a 3-factor SPQ-B solution accounted for $43 \%$ of the variance and generally converged with the cognitive-perceptual, interpersonal, and disorganized scales. Aycicegi and associates (2005) found a two-factor solution, reflecting negative symptoms and positive-type schizotypal features (with the disorganized items loading on these two factors), in undergraduate students in Turkey and the United States. Mata and coworkers (2006) showed that a 3-factor solution, resembling the three subscales, accounted for $35 \%$ of the variance in a sample of undergraduate students in Spain. The only confirmatory factor analysis published to date involved first-degree relatives of patients with schizophrenia and non-psychiatric controls (Compton et al., 2007) and indicated that although the 3-factor solution yielded reasonably good fit to the data, the single-factor solution provided equal fit.

Several potential methodological limitations of the present analysis should be considered. First, it is possible that specific demographic characteristics of this undergraduate convenience sample could have influenced these findings, possibly limiting generalizability. As noted, mean total and subscale scores in the present sample were lower than those reported in the undergraduate sample studied originally by Raine and Benishay (1995). This may suggest 
demographic, psychosocial, or reporting differences across otherwise seemingly similar undergraduate samples. Second, the 22 items of the SPQ-B were not measured in isolation but rather as items embedded within the larger 74-item SPQ. Despite these limitations, the findings indicate that further investigation of the factorial validity of the SPQ-B is warranted before the three proposed subscales are presumed to have factorial validity. Additional empirical studies using equally large, though perhaps more demographically diverse, samples could be used to create a more robust, albeit conceivably still abbreviated version of the SPQ.

Although the total SPQ-B score may provide useful information in terms of an efficient preliminary assessment of an individual's level of schizotypy, valid measurement of separable dimensions of the schizotypy construct may require a greater number of items than the three short subscales of the SPQ-B provide. Due to the complexity of the multi-dimensional schizotypy construct, it could be that factorial validity requires a full-version instrument rather than a very brief (e.g., 2-minute) version. Although Raine and Benishay (1995) selected the most reliable SPQ items and chose enough to ensure adequate internal reliability (e.g., three items from the both of the SPQ disorganized subscales were selected for a total of six items in the SPQ-B disorganized subscale), adequate internal reliability may not guarantee acceptable factorial validity.

As recommended previously (Compton et al., 2007), the total SPQ-B score and any subscale scores derived from the SPQ-B items should be assessed in individual samples before making assumptions about their psychometric properties. As in the previous report (involving 61 biological relatives of patients with schizophrenia and other psychotic disorders, as well as 57 non-psychiatric controls), the present study, conducted in a much larger sample of young adult undergraduate students, also suggests caution with respect to the proposed SPQ-B subscales. 
Ongoing research is required to determine whether or not specific dimensions of the multidimensional schizotypy construct can be measured adequately with a short series of self-report items rather than longer instruments. 


\section{References}

American Psychiatric Association, 1980. Diagnostic and Statistical Manual of Mental Disorders, Third Edition, Revised. Washington, DC: American Psychiatric Association.

Axelrod, S.R., Grilo, C.M., Sanislow, C., McGlashan, T.H., 2001. Schizotypal Personality Questionnaire-Brief: factor structure and convergent validity in inpatient adolescents. J. Personality Disord. 15, 168-179.

Aycicegi, A., Dinn, W.M., Harris, C.L., 2005. Validation of Turkish and English Versions of the Schizotypal Personality Questionnaire-B. Eur. J. Psycholog. Assess. 21, 34-43.

Bailey, E.L., Swallow, B.L., 2004. The relationship between cannabis use and schizotypal symptoms. Eur. Psychiatry. 19, 113-114.

Bedwell, J.S., Donnelly, R.S., 2005. Schizotypal personality disorder or prodromal symptoms of schizophrenia? Schizophr. Res. 80, 263-269.

Bedwell, J.S., Kamath, V., Baksh, E., 2006. Comparison of three computer-administered cognitive tasks as putative endophenotypes of schizophrenia. Schizophr. Res. 88, 36-46.

Compton, M.T., Chien, V.H., Bollini, A.M., 2007. Psychometric properties of the Brief Version of the Schizotypal Personality Questionnaire in relatives of patients with schizophreniaspectrum disorders and non-psychiatric controls. Schizophr. Res. 91, 122-131.

Compton, M.T., Goulding, S.M., Bakeman, R., McClure-Tone, E.B., 2009. Confirmation of a four-factor structure of the Schizotypal Personality Questionnaire among undergraduate students. Schizophr. Res. (in press).

Coryell, W.H., Zimmerman, M., 1989. Personality disorder in the families of depressed, schizophrenic, and never-ill probands. Am. J. Psychiatry. 146, 496-502. 
Dickey, C.C., McCarley, R.W., Niznikiewicz, M.A., Voglmaier, M.A., Seidman, L.J., Kim, S., Shenton, M., 2005. Clinical, cognitive, and social characteristics of a sample of neuroleptic-naive persons with schizotypal personality disorder. Schizophr. Res. 78, 297308.

Gilvarry, CM., Russell, A., Hemsley, D., Murray, R.M., 2001. Neuropsychological performance and spectrum personality traits in the relatives of patients with schizophrenia and affective psychosis. Psychiatr. Res. 101, 89-100.

Gooding, D.C., Matts, C.W., Rollmann, E.A., 2006. Sustained attention deficits in relation to psychometrically identified schizotypy: evaluating a potential endophenotypic marker. Schizophr. Res. 82, 27-37.

Houran, J., Irwin, H.J., Lange, R., 2001. Clinical relevance of the two-factor rasch version of the revised paranormal belief scale. Personality Individ. Diff. 31, 371-382.

Jahshan, C.S., Sergi, M.J., 2007. Theory of mind, neurocognition, and functional status in schizotypy. Schizophr. Res. 89, 278-286.

Kendler, K.S., McGuire, M., Gruenberg, A.M., Walsh, D., 1995. Schizotypal symptoms and signs in the Roscommon Family Study: their factor structure and familial relationship with psychotic and affective disorders. Arch. Gen. Psychiatry 52, 296-303.

Kremen, W.S., Faraone, S.V., Toomey, R., Seidman, L.J., Tsuang, M.T., 1998. Sex differences in self-reported schizotypal traits in relatives of schizophrenic probands. Schizophr. Res. 34, 27-37.

Mata, I., Gilvarry, C.M., Jones, P.B., Lewis, S.W., Murray, R.M., Sham, P.C., 2003. Schizotypal Personality Traits in Nonpsychotic Relatives Are Associated With Positive Symptoms in Psychotic Probands. Schizophr. Bull. 29, 273-283. 
Mata, I., Mataix-Cols, D., Peralta, V., 2005. Schizotypal Personality Questionnaire-Brief: factor structure and influence of sex and age in a nonclinical population. Personality Individ. Diff. 38, 1183-1192.

Miller, P., Byrne, M., Hodges, A., Lawrie, S.M., Owens, D.G., Johnstone, E.C., 2002. Schizotypal components in people at high risk of developing schizophrenia: early findings from the Edinburgh High-Risk Study. Br. J. Psychiatry. 180, 179-184.

Raine, A., 1991. The SPQ: a scale for the assessment of schizotypal personality based on DSMIII-R criteria. Schizophr. Bull. 17, 555-564.

Raine, A., Benishay, D., 1995. The SPQ-B: a brief screening instrument for schizotypal personality disorder. J. Personality Disord. 9, 346-355.

Raine, A., Reynolds, C., Lencz, T., Scerbo, A., Triphon, N., Kim, D., 1994. Cognitiveperceptual, interpersonal, and disorganized features of schizotypal personality. Schizophr. Bull. 20, 191-201.

Schiffman, J., Nakamura, B., Earleywine, M., LaBire, J., 2005. Symptoms of schizotypy precede cannabis use. Psychiatry Res. 134, 37-42.

Squires-Wheeler, E., Skodal, A.E., Friedman, D., Erlenmeyer-Kimling, L., 1988. The specificity of DSM-III schizotypal personality traits. Psychol. Med. 18, 757-765.

Squires-Wheeler, E., Skodal, A.E., Bassett, A., Erlenmeyer-Kimling, L., 1989. DSM-III-R schizotypal personality traits in offspring of schizophrenic disorder, affective disorder, and normal control parents. J. Psychiatr Res. 23, 229-239.

Tyrka, A.R., Cannon, T.D., Haslam, N., Mednick, S.A., Schulsinger, F., Schulsinger, H., Parnas, J., 1995. The latent structure of schizotypy: I. premorbid indicators of a taxon of 
individuals at risk for schizophrenia-spectrum disorders. J. Abnorm. Psychol. 104, 173183. 
Table 1. Fit Indices for the 3-Factor and Single-Factor Models of the 22-Item SPQ-B

\begin{tabular}{lrrrrrrr}
\hline Model & $\chi^{2}$ model & $d f_{\text {model }}$ & $\chi^{2}$ normed $^{\mathrm{a}}$ & RMSEA $^{\mathrm{b}}$ & $(90 \% \mathrm{CI})^{\mathrm{c}}$ & $\mathrm{SRMR}^{\mathrm{d}}$ & $\mathrm{CFI}^{\mathrm{e}}$ \\
\hline 3-factor & 878 & 206 & 4.26 & .068 & $(.064-.073)$ & .064 & .82 \\
single-factor & 1777 & 209 & 8.50 & .095 & $(.091-.100)$ & .074 & .70 \\
\hline
\end{tabular}

${ }^{\mathrm{a}} \chi^{2}$ model $/ d f_{\text {model }} ;{ }^{\mathrm{b}}$ root mean square error of approximation; ${ }^{\mathrm{c}} 90 \%$ confidence interval for the RMSEA; ${ }^{\mathrm{d}}$ standardized root mean square residual; ${ }^{\mathrm{e}}$ Bentler comparative fit index 